\title{
A retrograde apoptotic signal originating in NGF-deprived distal axons of rat sympathetic neurons in compartmented cultures
}

\author{
Sue-Ann Mok ${ }^{1}$, Karen Lund ${ }^{1}$, Robert B Campenot ${ }^{1}$ \\ ${ }^{1}$ Department of Cell Biology, University of Alberta, 5-14 Medical Sciences Building, Edmonton, Alberta, Canada T6G 2H7
}

Previous investigations of retrograde survival signaling by nerve growth factor (NGF) and other neurotrophins have supported diverse mechanisms, but all proposed mechanisms have in common the generation of survival signals retrogradely transmitted to the neuronal cell bodies. We report the finding of a retrograde apoptotic signal in axons that is suppressed by local NGF signaling. NGF withdrawal from distal axons alone was sufficient to activate the pro-apoptotic transcription factor, c-jun, in the cell bodies. Providing NGF directly to cell bodies, thereby restoring a source of NGF-induced survival signals, could not prevent $c$-jun activation caused by NGF withdrawal from the distal axons. This is evidence that c-jun is not activated due to loss of survival signals at the cell bodies. Moreover, blocking axonal transport with colchicine inhibited c-jun activation caused by NGF deprivation suggesting that a retrogradely transported pro-apoptotic signal, rather than loss of a retrogradely transported survival signal, caused c-jun activation. Additional experiments showed that activation of c-jun, pro-caspase-3 cleavage, and apoptosis were blocked by the protein kinase $C$ inhibitors, rottlerin and chelerythrine, only when applied to distal axons suggesting that they block the axon-specific pro-apoptotic signal. The rottlerin-sensitive mechanism was found to regulate glycogen synthase kinase 3 (GSK3) activity. The effect of siRNA knockdown, and pharmacological inhibition of GSK3 suggests that GSK3 is required for apoptosis caused by NGF deprivation and may function as a retrograde carrier of the axon apoptotic signal. The existence of a retrograde death signaling system in axons that is suppressed by neurotrophins has broad implications for neurodevelopment and for discovering treatments for neurodegenerative diseases and neurotrauma.

Keywords: nerve growth factor, apoptosis, retrograde apoptotic signal, sympathetic neuron, axon, glycogen synthase kinase 3, c-jun

Cell Research (2009) 19:546-560. doi: 10.1038/cr.2009.11; published online 3 February 2009

\section{Introduction}

During development, neurotrophin-responsive neurons extend their axons to innervate target cells releasing neurotrophins. Neurotrophins bind to cognate receptors on

Correspondence: Robert B Campenot

Tel: +1-780-492-7180; Fax: +1-780-492-0450

E-mail: bob.campenot@ualberta.ca

Abbreviations: NGF (nerve growth factor); PKC (protein kinase C); DAx (distal axons); CB/PAx (cell bodies/proximal axons); IAx (Intermediate axons); GSK3 (glycogen synthase kinase 3)

Received 8 October 2008; revised 10 October 2008; accepted 20 November 2008; published online 3 February 2009 axon terminals activating mechanisms supporting neuronal survival and differentiation. It is generally believed that target cells regulate their innervation by releasing neurotrophins in limiting amounts during development, and only neurons obtaining sufficient neurotrophin signals survive [1]. This process is a major contributor to the programmed cell death that eliminates about $50 \%$ of neurons during normal development [2].

The prototypical neurotrophin, nerve growth factor (NGF), elicits many of its biological effects through activation of the TrkA receptor [3]. It has been known for over 30 years that NGF applied to sympathetic axon terminals in vivo is retrogradely transported along the axons to the cell bodies [4], suggesting that NGF bound to its 
receptor and internalized at the nerve terminal is transported to the cell bodies where it generates its survival effect [5]. Subsequent studies providing evidence for retrograde transport of phosphorylated TrkA [6] and the cooccurrence of NGF and TrkA in endosomal preparations [7] have supported this view, now known as the "signaling endosome hypothesis" $[8,9]$.

However, evidence from our laboratory indicates that signals initiated by NGF applied to distal axons of rat sympathetic neurons in compartmented cultures support neuronal survival without transport of NGF to the cell bodies [10] and with no requirement for the appearance of phosphorylated TrkA in the cell bodies [11, 12]. These data suggest that phosphorylated TrkA in distal axons activates downstream signaling mechanisms in the distal axons that communicate to the cell bodies. Here, we present evidence for a fundamentally different retrograde survival mechanism in which NGF at distal axons suppresses an apoptotic signaling mechanism in distal axons. This axon apoptotic signal, when derepressed in response to NGF withdrawal, sends a pro-apoptotic signal retrogradely to the cell body to promote the apoptotic program.

\section{Results}

For these investigations neurons were plated in compartmented cultures and maintained for 5-7 days with 10

A

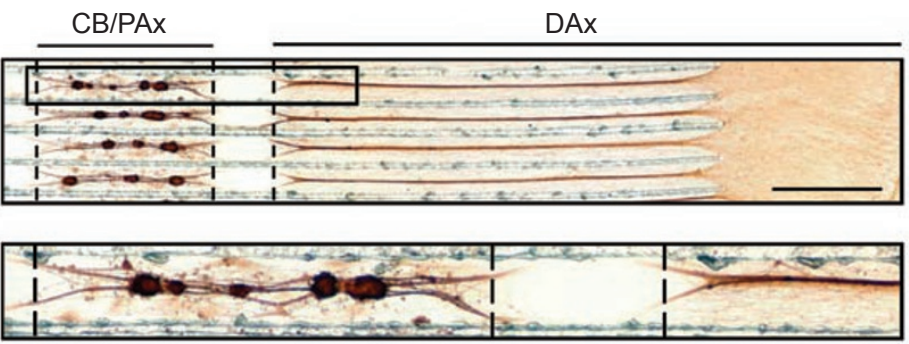

B

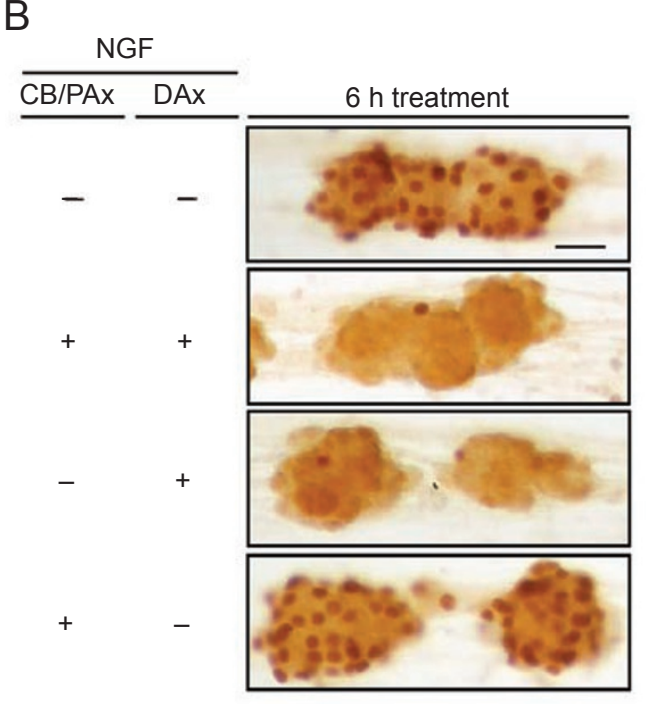

D

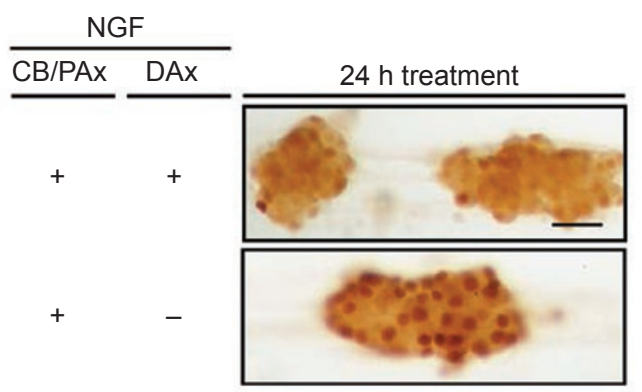

C

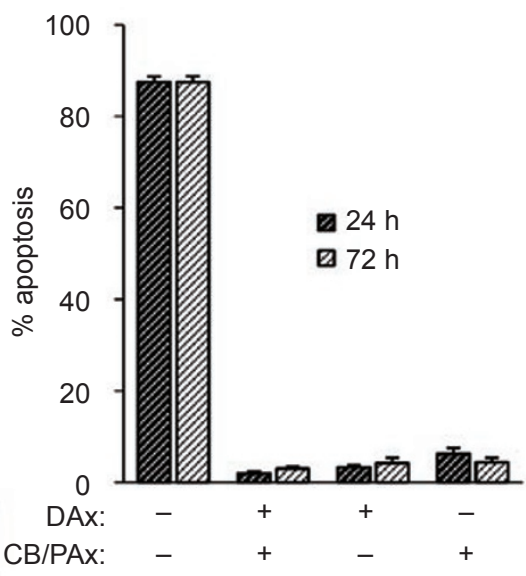

E

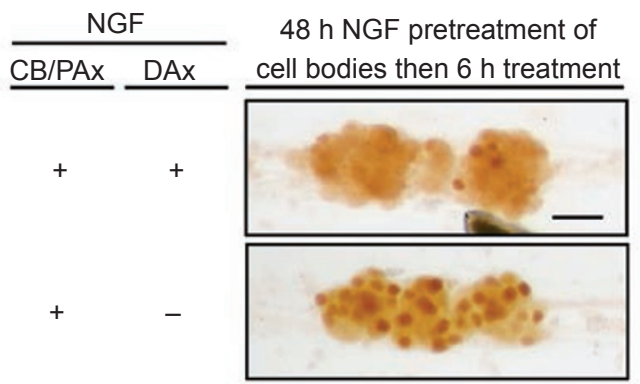


ng/ml NGF supplied to the cell bodies and proximal axons (CB/PAx) and $50 \mathrm{ng} / \mathrm{ml}$ NGF supplied to distal axons (DAx) before initiating the experimental treatments (Figure 1A). To assess the effects of NGF in our experiments, $\mathrm{CB} / \mathrm{PAx}$ and/or DAx were given either media containing $50 \mathrm{ng} / \mathrm{ml} \mathrm{NGF}$ to maintain robust NGF signaling or media lacking NGF and instead supplied with $24 \mathrm{nM}$ NGF antibodies to terminate all NGF signaling. Activation of the apoptotic program in the cell bodies was assessed after $6 \mathrm{~h}$ of experimental treatment by monitoring the phosphorylation (Ser 63) of the pro-apoptotic transcription factor, c-jun, by immunocytochemistry or immunoblot. c-jun is activated early during the apoptosis of NGFdeprived sympathetic neurons [13] by phosphorylation of Ser 63 and 73 residues by JNKs [14, 15]. NGF withdrawal induces phosphorylation of c-jun and its translocation to the nucleus [16] where it promotes expression of proapoptotic proteins such as BIM [17]. Deletion of c-jun in sympathetic neurons prevents apoptosis in response to NGF withdrawal [18] indicating a requirement of cjun for NGF deprivation-induced apoptosis. Apoptosis was assayed by Hoechst staining of nuclei; an apoptosed neuron contained a condensed nucleus after $24 \mathrm{~h}$ and no nucleus after 48 or $72 \mathrm{~h}$ of treatment.

Local withdrawal of NGF from distal axons induces an apoptotic signal that activates c-jun in cell bodies

To assess how NGF supplied to DAx versus cell bodies affects the state of c-jun activation, $\mathrm{CB} / \mathrm{PAx}$ and/or DAx were supplied with NGF or deprived of NGF for 6 $\mathrm{h}$ and then assayed for nuclear accumulation of activated c-jun. NGF-deprived neurons displayed nuclear staining, but neurons supplied with NGF either in all compartments or only at their DAx, both conditions that support survival (see Figure 1C), displayed virtually no nuclear staining (Figure 1B). Unexpectedly, when NGF was supplied directly to $\mathrm{CB} / \mathrm{PAx}$ but removed from DAx, nuclear accumulation of phosphorylated c-jun was observed, similar to cultures completely deprived of NGF (Figure 1B), but the neurons did not undergo apoptosis for at least $72 \mathrm{~h}$, the longest time observed (Figure 1C). Nuclear staining persisted for at least $24 \mathrm{~h}$ in neurons with NGF-deprived DAx (Figure 1D). Neurons pretreated for $48 \mathrm{~h}$ with $50 \mathrm{ng} / \mathrm{ml} \mathrm{NGF}$ in all compartments, to ensure that survival signaling mechanisms in the cell bodies were fully stimulated, still displayed nuclear accumulation of phosphorylated c-jun in response to withdrawal of NGF from their DAx (Figure 1E). In light of these results, we hypothesize that the loss of NGF signaling in DAx generates an apoptotic signal in the axons that travels retrograde to the cell bodies to produce c-jun activation. We further hypothesize that survival mechanisms activated by NGF supplied to the CB/PAx cannot deactivate the retrograde apoptotic signal that activates c-jun, and instead support survival by acting downstream of cjun to counteract the apoptotic signal arriving from DAx.

\section{The axonal apoptotic signal requires microtubule-based transport in axons \\ NGF is retrogradely transported [19] along microtu- bules in a dynein-dependent process [20] and was there- fore used as a marker of microtubule-based retrograde}

Figure 1 Sustained activation of the pro-apoptotic molecule, c-jun, is induced by local NGF withdrawal at the DAx even when NGF survival signals are supplied directly to the cell bodies. (A) Schematic diagram of a compartmented culture and images of sympathetic neurons in a 1-week-old culture immunostained for tubulin. The schematic diagram (left) shows a Teflon divider that partitions a 35-mm tissue culture dish (dish not shown) into compartments that separate the media supplied to the cell bodies/proximal axons (CB/PAx) from media supplied to the distal axons (DAx) of sympathetic neurons cultured from newborn rats. The divider is applied to the dish using a thin layer of silicone grease. Dissociated neurons are plated in the center compartment, and axons extend left and right under the barriers and into the distal compartments. The upper right panel shows the CB/PAx in the center compartment and DAx in the right compartment in four tracks, out of a total of 16, in a compartmented culture. The scale bar equals $1 \mathrm{~mm}$. The lower right panel is a higher magnification of the part of a single track boxed in the top panel. Dotted lines indicate barrier boundaries. Axons under the barriers are unstained. (B) C-jun is activated when NGF is removed from DAx. Compartments were supplied with $50 \mathrm{ng} / \mathrm{ml} \mathrm{NGF} \mathrm{(+)} \mathrm{or} \mathrm{media} \mathrm{lacking} \mathrm{NGF} \mathrm{and} \mathrm{containing}$ NGF antibodies (-) as indicated for $6 \mathrm{~h}$. Following treatment, CB/PAx were processed for immunocytochemistry with an antibody recognizing phosphorylated c-jun (Ser 63). c-jun activation is characterized by nuclear accumulation of phosphorylated c-jun. The scale bar equals $50 \mu \mathrm{m}$. Results are representative of three independent experiments. (C) Local withdrawal of NGF from DAx does not result in apoptosis if NGF is provided to the CB/PAx. NGF (+) or NGF antibodies (-) were supplied as indicated for 24 or $72 \mathrm{~h}$. Quantification of apoptosis is the percentage of neurons with condensed nuclei per group. At 72 $\mathrm{h}$, neurons lacking nuclear staining were also included in the count (see Materials and Methods). A minimum of 250 neurons were assessed per culture. Data were compiled from two experiments ( \pm SEM, $n=6$ cultures). (D, E) Cultures were treated and analyzed as in (B). Results are representative of three independent experiments. The scale bar equals $50 \mu \mathrm{m}$. (D) C-jun activation following 24-h treatment. (E) CB/PAx were pretreated for $48 \mathrm{~h}$ with $50 \mathrm{ng} / \mathrm{ml} \mathrm{NGF}$ in all compartments prior to experimental treatment. 
transport. The retrograde transport of Cy3-labeled NGF applied to DAx was abolished by the application of the microtubule-destabilizing drug, colchicine $(10 \mu \mathrm{M})$, to DAx (Figure 2A). Colchicine applied to DAx of NGFdeprived neurons substantially inhibited the activation of $\mathrm{c}$-jun in $\mathrm{CB} / \mathrm{PAx}$ (Figure 2B) suggesting that c-jun is activated by a retrograde death signal from DAx rather than by the loss of a retrograde survival signal from DAx. Colchicine application to DAx in the presence of NGF, on its own, produced a low level of c-jun activation simi-

\section{A}



B

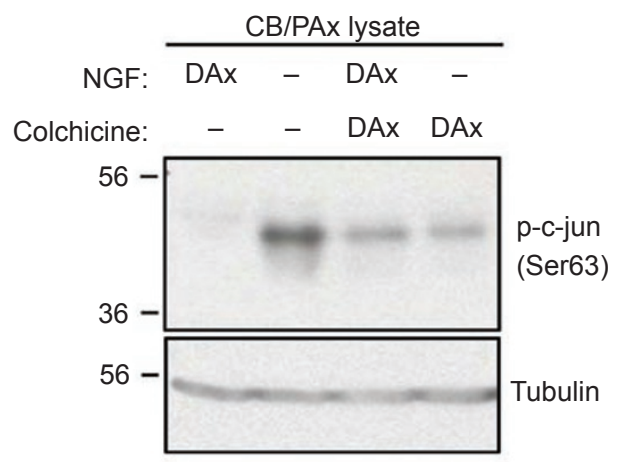

Figure 2 Colchicine applied to distal axons (DAx) during NGF withdrawal blocks activation of c-jun in the cell bodies. (A) Colchicine blocks retrograde transport of Cy3-labeled NGF. Cy3-labeled NGF (50 ng/ml) was applied to DAx for $6 \mathrm{~h}$. Colchicine $(10 \mu \mathrm{M})$ or 20 -fold excess unlabeled NGF $(1 \mu \mathrm{g} / \mathrm{ml})$ was also supplied to DAx during treatment as indicated. Cell bodies/ proximal axons (CB/PAx) were monitored for the accumulation of Cy3-labeled NGF. Results are representative of three independent experiments. (B) Colchicine applied to DAx blocks c-jun activation during NGF withdrawal. DAx were supplied with $50 \mathrm{ng} / \mathrm{ml} \mathrm{NGF}$ or NGF antibodies (-) in the absence or presence of $10 \mu \mathrm{M}$ colchicine where indicated. CB/PAx in all cultures were treated with NGF antibodies. Following a $6 \mathrm{~h}$ treatment, CB/PAx lysates were analyzed by immunoblotting with an antibody recognizing phosphorylated c-jun (Ser 63). The blot was reprobed for $\beta$-tubulin as a loading control. Results are representative of four independent experiments. lar to that observed in colchicine-treated, NGF-deprived neurons suggesting that this slight activation may be a side effect of colchicine.

We attempted to determine if blocking axonal transport would prevent apoptosis during NGF deprivation. Unfortunately, colchicine application resulted in the degradation of DAx starting at $12 \mathrm{~h}$ (data not shown). Therefore, experiments measuring neuronal survival after $24 \mathrm{~h}$ of colchicine treatment were not feasible.

Rottlerin and chelerythrine block the retrograde apoptotic signal only when applied to distal axons

While screening a variety of compounds (data not shown), we found that rottlerin $(2.5 \mu \mathrm{M})$ almost completely prevented nuclear accumulation of phosphorylated c-jun only when applied to DAx (Figure 3A and 3B). Rottlerin did not itself cause c-jun activation when applied either at the DAx or the CB/PAx of cultures maintained with NGF in all compartments.

Rottlerin was originally characterized as a protein kinase $\mathrm{C}$ (PKC) inhibitor with specificity for the novel $\mathrm{PKC}$ isoform delta $(\mathrm{PKC} \delta, \mathrm{IC} 50=3-6 \mu \mathrm{M}$ in rat brain) over other PKC isoforms [21]. We compared the effects of rottlerin with the effects of two other PKC inhibitors with specificities for different PKC isoforms: chelerythrine, a general PKC inhibitor [22] and Gö6976, an inhibitor of conventional PKC isoforms but not novel PKC isoforms such as $\mathrm{PKC} \delta$ [23]. The indicated treatments (Figure 4A) were applied to NGF-deprived neurons for $24 \mathrm{~h}$ after which neurons were assayed for apoptosis.

NGF-deprived neurons (black bars) without drug treatment underwent $84 \%$ apoptosis, but only $32 \%$ and $48 \%$ with rottlerin $(2.5 \mu \mathrm{M})$ or chelerythrine $(10 \mu \mathrm{M})$ respectively supplied to DAx. Neither drugs prevented apoptosis when applied to CB/PAx. Chelerythrine applied to DAx, like rottlerin, prevented c-jun activation in NGF-deprived neurons (Supplementary information, Figure S1). Rottlerin's protection lasted for at least $72 \mathrm{~h}$ (Figure 4B). Gö6976 was not protective from either site of application (Figure 4A). Since PKC $\delta$ is a target of both rottlerin and chelerythrine, but not Gö6976, these results initially suggested that $\mathrm{PKC} \delta$ might be required for generating the apoptotic signal in axons, but see below.

The cleavage of pro-caspase-3 to caspase- 3 in the cell bodies/proximal axons is blocked by rottlerin only when applied to distal axons

Cleavage of pro-caspase- 3 to its active form, caspase-3, is a required step occurring late in sympathetic neuron apoptosis [24]. To determine if pro-caspase-3 cleavage requires the apoptotic signal from DAx, sympa- 

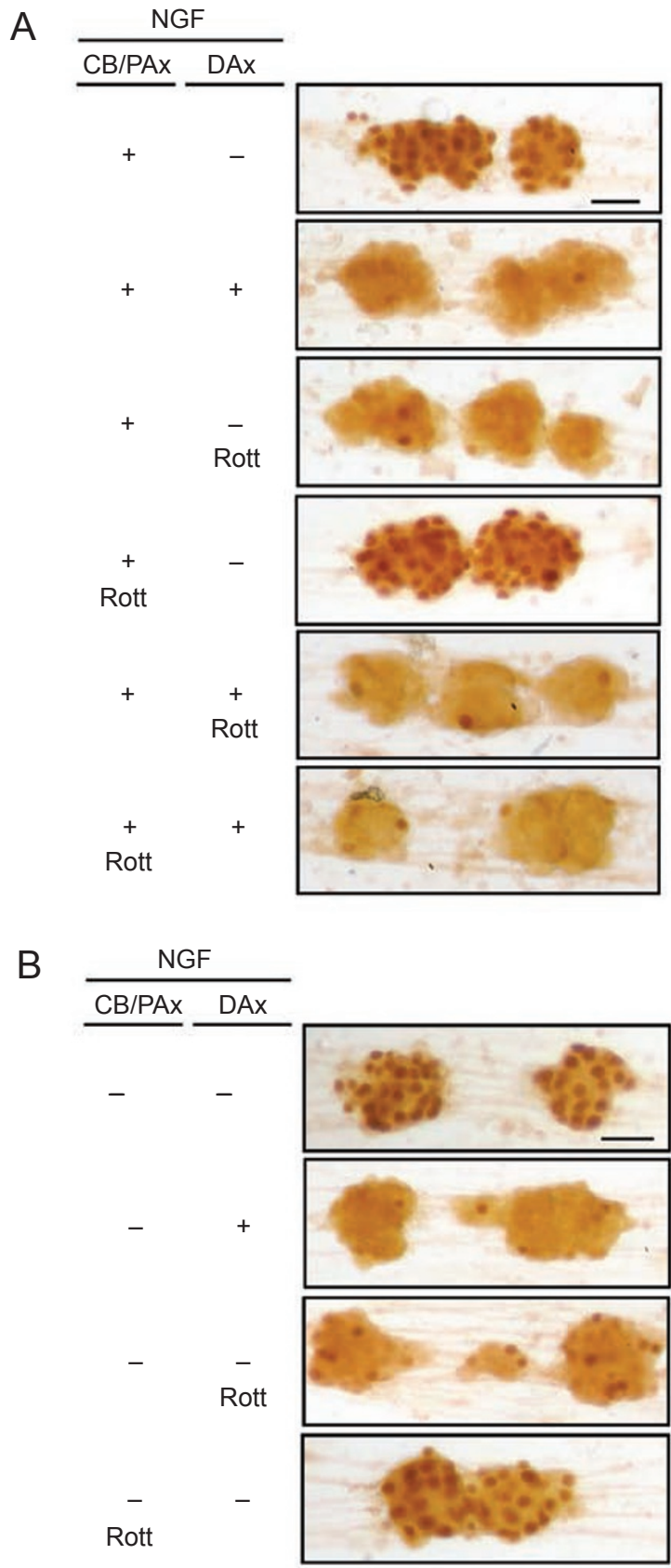

Figure 3 Rottlerin acts exclusively on distal axons (DAx) to prevent activation of pro-apoptotic c-jun. Compartmented cultures were supplied with $50 \mathrm{ng} / \mathrm{ml} \mathrm{NGF} \mathrm{(+)} \mathrm{or} \mathrm{media} \mathrm{lacking} \mathrm{NGF} \mathrm{and}$ containing NGF antibodies (-) in the presence or absence of rottlerin (Rott, $2.5 \mu \mathrm{M}$ ) to the cell bodies/proximal axons (CB/ $\mathrm{PAx}$ ) or the DAx as indicated for $6 \mathrm{~h}$. Then cultures were analyzed as in Figure 1B. The scale bar equals $50 \mu \mathrm{m}$. (A) Rottlerin when applied to DAx, but not when applied to CB/PAx, prevented c-jun activation caused by local withdrawal of NGF from DAx. Results are representative of three independent experiments. (B) Rottlerin when applied to DAx, but not when applied to CB/ PAx, prevented c-jun activation caused by complete withdrawal of NGF. Results are representative of four independent experiments. thetic neurons were deprived of NGF for $21 \mathrm{~h}$ with different distributions of rottlerin. Immunoblot analysis of lysates showed a $17 \mathrm{kDa}$ band corresponding to cleaved pro-caspase-3 appearing in $\mathrm{CB} / \mathrm{PAx}$, but not in DAx of NGF-deprived neurons (Figure 4C). Examination of $\mathrm{CB} / \mathrm{PAx}$ lysates showed that NGF deprivation greatly increased the intensity of the caspase- 3 band compared with control cultures maintained with NGF at their DAx (Figure 4C and 4D). Rottlerin prevented the increase in caspase-3 only when applied to DAx (Figure 4D).

\section{Rottlerin inhibits the axonal apoptotic signal at axon ter- minal segments}

Sympathetic neurons were grown in five-compartmented cultures in which the neurons extend their axons across one barrier, through a 1 -mm-wide intermediate compartment, and then across a second barrier into the distal compartment, extending a distance of greater than $2 \mathrm{~mm}$. After 5 days in culture, the intermediate axons (IAx) and CB/PAx were deprived of NGF for 2 days to eliminate neurons with axons that had not crossed into the distal axon compartment. Neurons were then treated with NGF on DAx or completely deprived of NGF in the presence or absence of rottlerin in the indicated compartments for $24 \mathrm{~h}$ and assessed for apoptosis (Figure 5). NGF deprivation caused $87 \%$ of neurons to undergo apoptosis. Apoptosis was reduced to $27 \%$ when rottlerin was supplied to DAx, but rottlerin applied to IAx did not provide significant protection (84\% apoptosis). Rottlerin applied to $\mathrm{CB} / \mathrm{PAx}$ offered a small but significant protection (74\% apoptosis). These results indicate that the axonal apoptotic signal blocked by rottlerin mainly originates in the distal axon segments and is retrogradely transported by downstream signaling molecules through the IAx to the cell bodies. This suggests that the axon terminals may be the site of origin of the retrograde apoptotic signal.

The axonal apoptotic signal is not sensitive to PKC knockdown by siRNA

$\mathrm{PKC} \delta$ has been implicated in mediating glutamate toxicity in primary cortical neurons [25] and mediating the effects of neurotoxins and oxidative stress in dopaminergic neurons [26]. Since rottlerin and chelerythrine both inhibit $\mathrm{PKC} \delta$, we investigated if $\mathrm{PKC} \delta$ activity was required to generate the retrograde apoptotic signal in DAx. To knock down the expression of $\mathrm{PKC} \delta$, neurons were transfected by nucleofection [27] with siRNAs targeting PKC $\delta$ mRNA, immediately prior to plating in mass cultures. Control neurons were alternatively transfected without siRNA (-) or with a negative control siRNA (Neg) designed to not match any known rat mRNA 
A

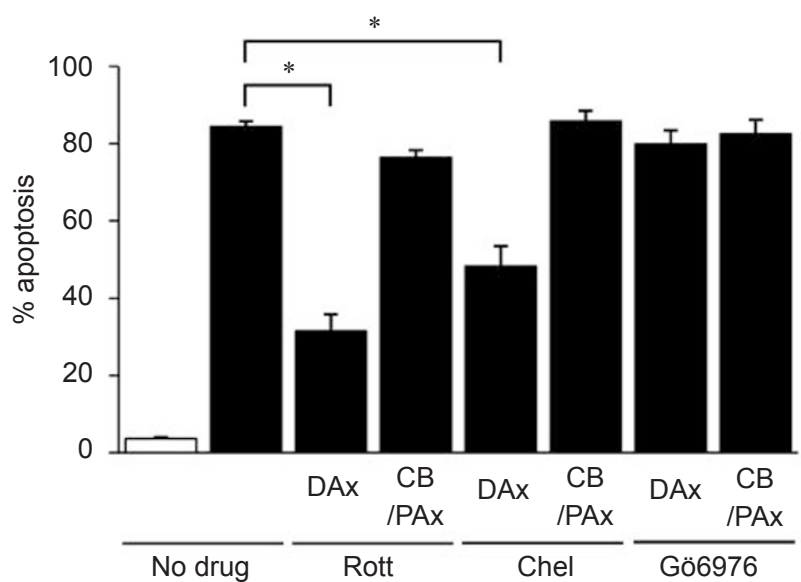

B

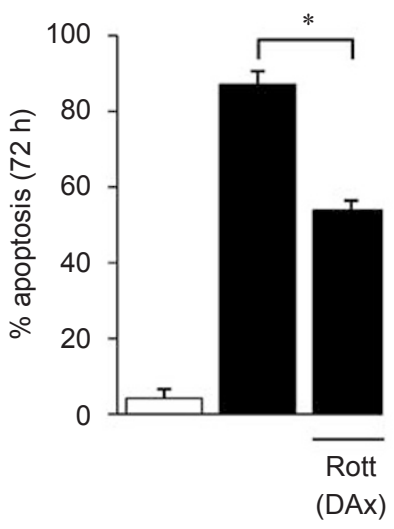

C

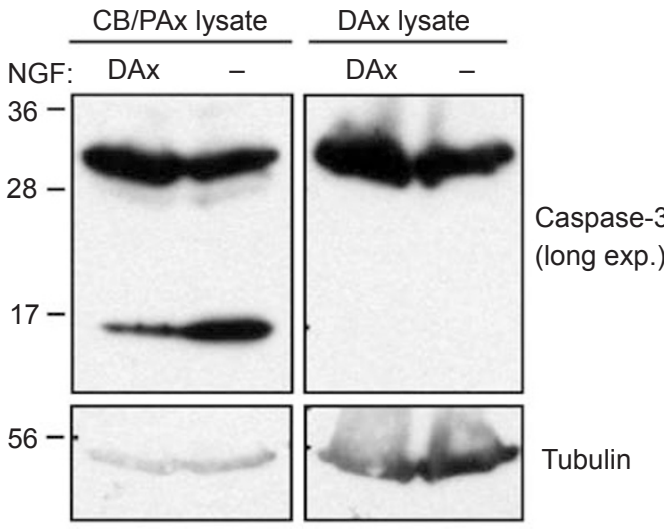

D

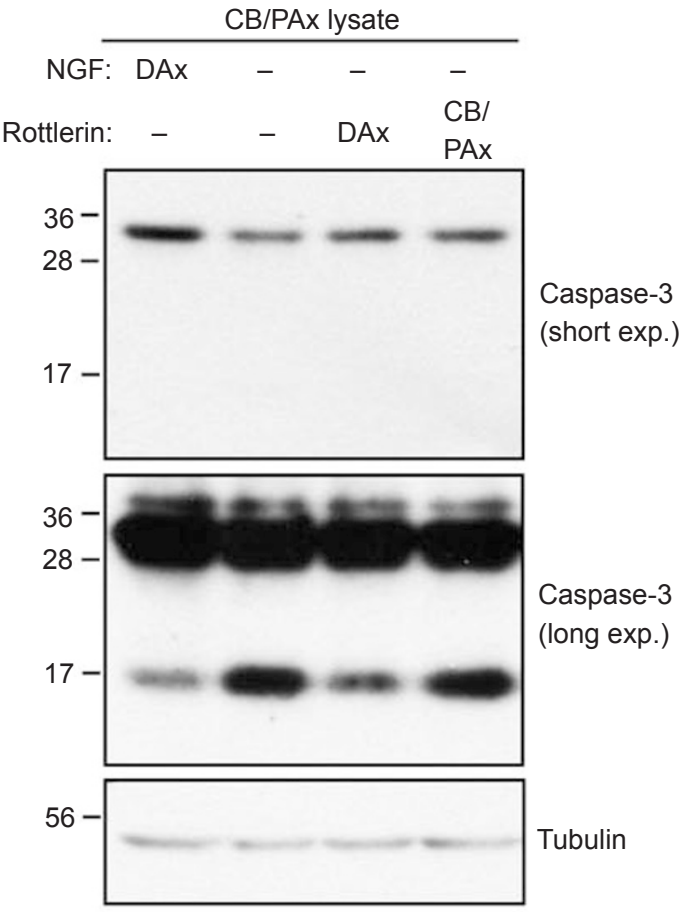

Figure 4 Rottlerin applied to distal axons (DAx) prevents apoptosis and pro-caspase-3 cleavage of sympathetic neurons subjected to complete NGF withdrawal. (A, B) Cell bodies/proximal axons (CB/PAx) of all cultures were given NGF antibodies while DAx were treated with either $50 \mathrm{ng} / \mathrm{ml}$ NGF (white bar) or NGF antibodies (black bars). Concurrently, the PKC inhibitors, rottlerin (Rott, $2.5 \mu \mathrm{M})$, chelerythrine (Chel, $10 \mu \mathrm{M})$, or Gö6976 $(25 \mathrm{nM})$ were supplied in the compartments indicated. Apoptotic neurons were visually identified by their condensed, fragmented nuclei or absence of nuclei as in Figure 1C. The percentage of apoptotic neurons in each group is plotted $( \pm$ SEM) from data compiled from three experiments. (A) Application of PKC $\delta$ inhibitors to DAx, but not to CB/PAx, inhibits apoptosis ( $n \geq 8$ cultures per group, ${ }^{*} P<0.001$, one-way ANOVA post-hoc Scheffe test). (B) Rottlerin application to DAx inhibits apoptosis of compartmented cultures deprived of NGF for $72 \mathrm{~h}$ ( $n \geq 5$ cultures per group, ${ }^{*} P<0.001$, one-way ANOVA post-hoc Scheffe test). (C and D) DAx were supplied with NGF (50 ng/ml) or with NGF antibodies (-) where indicated. CB/PAx were treated with NGF antibodies in all cultures. Rottlerin $(2.5 \mu \mathrm{M})$ was supplied in the specified compartments during treatment. Lysates were analyzed by immunoblot after $21 \mathrm{~h}$ of treatment. The blot was probed with an antibody recognizing the p35, full-length pro-caspase-3 and the p17 cleaved form. The cleaved form was only visualized after long exposure times. The blot was reprobed for $\beta$-tubulin as a loading control. Results are representative of three independent experiments. (C) Pro-caspase-3 cleavage occurs in the CB/PAx but not in DAx during NGF withdrawal. (D) Rottlerin application to DAx during NGF withdrawal decreases pro-caspase-3 cleavage. 



Figure 5 Rottlerin blocks the axonal apoptotic signal at axon terminals. Sympathetic neurons were cultured in five-compartmented cultures shown in the schematic diagram. After 5 days in culture, NGF was withdrawn from intermediate axons (IAx) and cell bodies/proximal axons (CB/PAx) for 2 days to remove neurons whose distal axons (DAx) had not crossed into the distal compartments. Following this pretreatment, CB/PAx and IAx were given medium containing NGF antibodies and DAx were treated with $50 \mathrm{ng} / \mathrm{ml}$ NGF (white bar), NGF antibodies (black bars), and rottlerin $(2.5 \mu \mathrm{M})$ where indicated for $24 \mathrm{~h}$. Apoptotic nuclei were assessed as in Figure 1C. Data plotted for each group are compiled from three experiments ( \pm SEM, $n \geq 7$ cultures, ${ }^{*} P<0.05$, $* * P<0.001$, one-way ANOVA post-hoc Scheffe test).

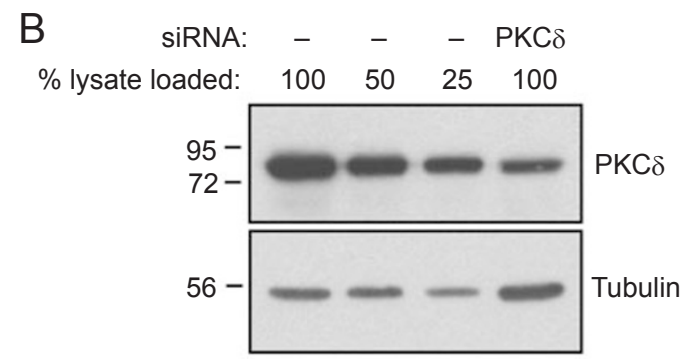

D

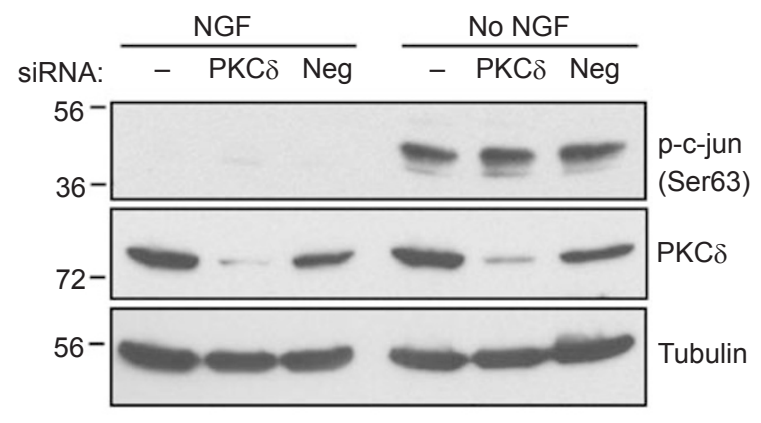

Figure 6 Knockdown of PKC $\delta$ by siRNA does not prevent NGF withdrawal-induced apoptosis of sympathetic neurons. Sympathetic neurons in mass cultures were transfected by nucleofection with a pool of four siRNAs (PKC $\delta$ ) targeting rat PKC $\delta$ mRNA prior to plating in mass culture. Transfections were also carried out with no siRNA (-) or a non-specific siRNA (Neg) as controls. (A) PKC $\delta$ knockdown at the protein level. Transfected neurons were harvested on day 3 or 6 in culture as indicated, and equivalent amounts of cell lysates $(8 \mu \mathrm{g})$ were analyzed by immunoblot. The blot was probed with an antibody against $\mathrm{PKC} \delta$ and probed for $\beta$-tubulin as a loading control. Results are representative of three independent experiments. (B) Quantitation of PKC $\delta$ knockdown. Day 4 cultures were harvested and loaded onto an SDS-PAGE gel in the relative amounts indicated $(8 \mu \mathrm{g}=100 \%)$. The blot was probed for $\mathrm{PKC} \delta$ and probed for $\beta$-tubulin as a loading control. Results are representative of two independent experiments. (C) Apoptosis in response to NGF withdrawal. Day 5 transfected cultures were maintained in NGF (white bars) or deprived of NGF (black bars) for $24 \mathrm{~h}$. Rottlerin $(2.5 \mu \mathrm{M})$ was supplied where indicated. Apoptosis was quantified as in Figure $1 \mathrm{C}$ as the percentage of neurons with condensed and fragmented nuclei per group ( \pm SEM, $n=4$ cultures, N.S.=not significant, one-way ANOVA post-hoc Tukey HSD). Data were compiled from two experiments. (D) c-jun activation. On day 3 in culture, neurons transfected as indicated, were maintained in NGF (NGF) or deprived of NGF (no NGF) for $6 \mathrm{~h}$, then harvested and analyzed by immunoblot. Blots were probed with an antibody recognizing phosphorylated c-jun (Ser 63). Blots were reprobed for PKC $\delta$ and $\beta$-tubulin. Results are representative of three independent experiments. 
sequences. Immunoblots of 3- and 6-day-old cultures showed a substantial reduction in $\mathrm{PKC} \delta$ protein levels in neurons transfected with $\mathrm{PKC} \delta$ siRNA (Figure 6A), estimated by dilution to be greater than $75 \%$ (Figure $6 \mathrm{~B}$ ). Transfecting neurons with $\mathrm{PKC} \delta$ siRNA did not prevent apoptosis (98\%) nor did it prevent rottlerin from rescuing NGF-deprived neurons, suggesting that rottlerin's molecular target was still present in neurons with reduced PKC $\delta$ (Figure 6C). Immunoblot analysis showed that knockdown of PKC $\delta$ did not prevent phosphorylation of c-jun during NGF deprivation (Figure 6D). PKC $\theta$ is another PKC isoform inhibited by rottlerin [28]. A cell permeable peptide inhibitor targeting this isoform did not prevent apoptosis induced by NGF withdrawal in mass cultures (Supplementary information, Figure S2). Furthermore, activation of PKCs by phorbol-12-myristate13-acetate (PMA) did not activate c-jun nor induce apoptosis in NGF-maintained cultures (Supplementary information, Figure S3). These results do not support a role for $\mathrm{PKC}$ in triggering the retrograde apoptotic signal.

\section{Rottlerin inhibits activation of GSK3, a kinase that pro- motes apoptosis of sympathetic neurons during NGF withdrawal}

In a further effort to identify rottlerin's protective mechanism, we examined the activation of potential pro-apoptotic molecules. One such molecule, glycogen synthase kinase 3 (GSK3) $\beta$, can function in neurons as a pro-apoptotic kinase [29]. GSK3 $\beta$ is also implicated in axon growth $[30,31]$ and Alzheimer's disease pathology [32]. GSK $3 \beta$ and its closely related isoform GSK $3 \alpha$ [33] are regulated by inhibitory phosphorylation (Ser 21 for GSK $3 \alpha$ and Ser 9 for GSK3 $\beta$ ) $[34,35]$. We analyzed the activation state of both GSK3 isoforms by immunoblot in NGF-deprived, rottlerin-treated neurons (Figure 7A). GSK $3 \alpha$ and GSK $3 \beta$ were activated (dephosphorylated) in DAx within $6 \mathrm{~h}$ of NGF deprivation (compare lanes 5 and 6). Loss of phosphorylation is not caused by decreases in the levels of total GSK3 (Figure 7A). Rottlerin applied to DAx partially blocked the activation of both isoforms in DAx (lane 7), while rottlerin applied to CB/ PAx did not prevent activation in DAx (lane 8), suggesting that GSK3 is activated in NGF-deprived DAx by an upstream pathway sensitive to rottlerin.

We next determined whether GSK3 activity promoted apoptosis of NGF-deprived sympathetic neurons. Two inhibitors of GSK3, lithium chloride $(\mathrm{LiCl})$ and kenpaullone [36], block GSK $3 \alpha$ and GSK3 $\beta$ activity. Application of $\mathrm{LiCl}$ to DAx or $\mathrm{CB} / \mathrm{PAx}$ during NGF withdrawal (Figure 7B) significantly reduced apoptosis $(49 \%$ and $46 \%$ apoptosis, respectively). Application of $\mathrm{LiCl}$ to the entire neuron also reduced apoptosis to $41 \%$, but not sig- nificantly greater than application to either compartment alone, indicating that the protective effects were not additive. Application of kenpaullone (Figure 7C) to DAx and $\mathrm{CB} / \mathrm{PAx}$ inhibited apoptosis to $51 \%$ and $54 \%$, respectively. However, application of kenpaullone to all compartments (Figure 7C) resulted in a greater protection $(33 \%$ apoptosis) than either compartment alone suggesting an additive effect. The results with $\mathrm{LiCl}$ and kenpaullone suggest that GSK3 activity contributes to apoptosis in response to NGF deprivation. Moreover, the finding that these inhibitors afford protection when applied to DAx or $\mathrm{CB} / \mathrm{PAx}$ is consistent with the concept that GSK3 plays a role in carrying the retrograde apoptotic signal from DAx to the cell bodies, or that GSK3 activity is required in both DAx and the cell bodies to promote apoptosis. Note that rottlerin applied to DAx may have produced a slight increase in the phosphorylation of GSK3 in CB/ PAx (Figure 7A, compare lanes 2 and 3), but this effect was small and is difficult to interpret (see Discussion).

Consistent with possible roles for both GSK3 $\alpha$ and GSK3 $\beta$ in retrograde apoptotic signaling, siRNA knockdown of either isoform did not prevent apoptosis of NGF-deprived sympathetic neurons (Supplementary information, Figure S4). However, partial (75\% or greater) knockdown of both as determined by immunoblot caused a $27 \%$ reduction in apoptosis relative to negative control transfected neurons (Figure 8A and 8B). The incomplete knockdown of GSK3 isoforms may account for the smaller decrease in apoptosis compared with that observed in the GSK3 inhibitor experiments.

\section{Discussion}

Previous explanations of how NGF and other neurotrophins promote neuronal survival assume that NGF supports neuronal survival by initiating survival signals at axon terminals that are transmitted retrogradely to the cell bodies [8, 9, 37]. In contrast, our results suggest that NGF at the distal axons also supports survival by suppressing a mechanism in the axons, which in the absence of NGF signaling, produces a retrogradely transported pro-apoptotic signal. This signal is transported to the cell bodies where it initiates the apoptotic program. To obtain this evidence we used two indicators of apoptosis: (1) apoptosis itself as indicated by chromatin condensation and loss of chromatin staining, which are evident at $24 \mathrm{~h}$ after NGF withdrawal; and (2) nuclear accumulation of activated c-jun, which occurs within $6 \mathrm{~h}$ of NGF withdrawal. c-jun activation was a critical indicator of the initiation of apoptosis in our studies. Several studies from other laboratories have demonstrated that c-jun is required for apoptosis of sympathetic neurons during 
A

\begin{tabular}{|c|c|c|c|c|c|c|c|}
\hline \multicolumn{4}{|c|}{ CB/PAx lysate } & \multicolumn{4}{|c|}{ DAx lysate } \\
\hline NGF: & - & - & - & DAx & - & - & - \\
\hline ott: & - & DAx & $\begin{array}{c}\text { CB } \\
\text { /PAx }\end{array}$ & - & - & DAx & $\begin{array}{c}\mathrm{CB} \\
/ \mathrm{PAx}\end{array}$ \\
\hline
\end{tabular}

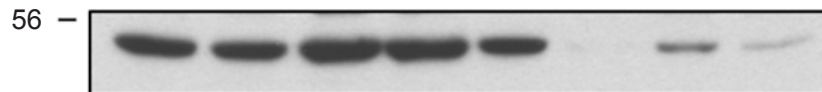

pGSK3 $\alpha($ Ser21)

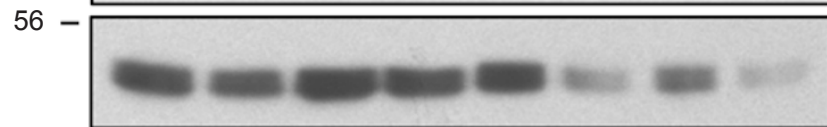

pGSK3 $\beta$ (Ser9)

56

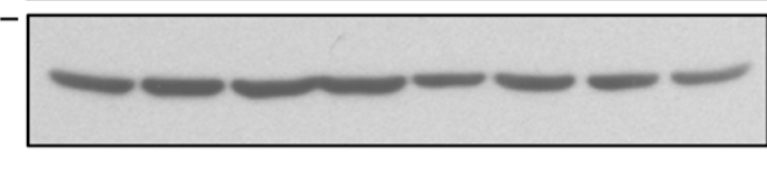

Actin

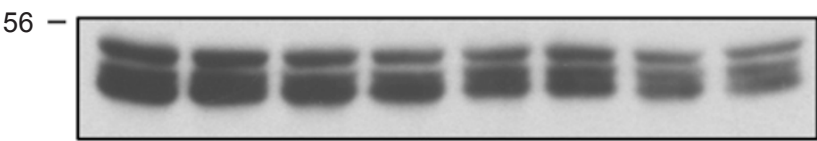

Total GSK3

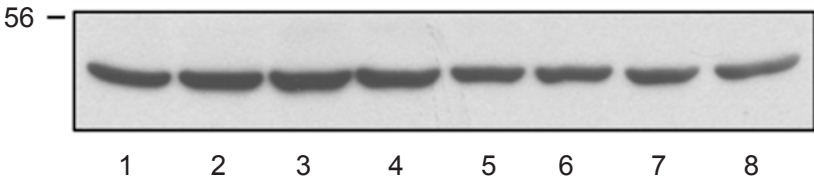

Actin

B

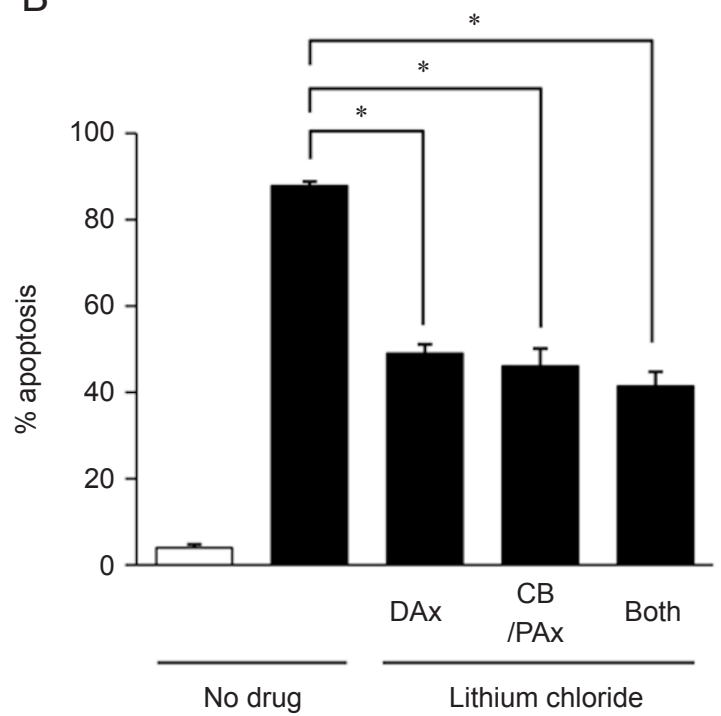

C

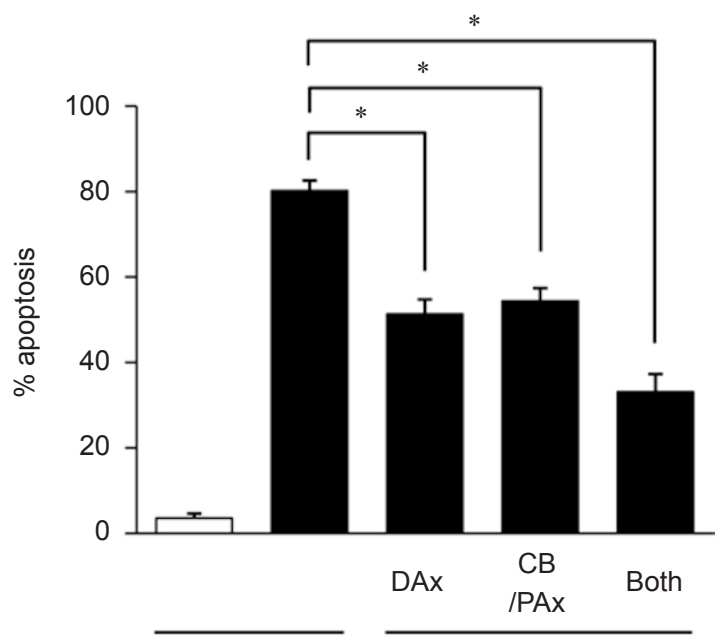

No drug

Kenpaullone

Figure 7 Rottlerin inhibits activation of GSK3, a kinase required for apoptosis in response to NGF withdrawal. (A) Rottlerin inhibits activation (dephosphorylation) of GSK3 $\alpha$ and GSK3 3 . Distal axons (DAx) were treated with $50 \mathrm{ng} / \mathrm{ml}$ NGF or NGF antibodies (-). Cell bodies/proximal axons (CB/PAx) were treated with NGF antibodies in all cultures. Rottlerin (Rott) was supplied to compartments as indicated. Lysates from each group were split into two equal samples and analyzed on separate immunoblots following $6 \mathrm{~h}$ of treatment. One blot was probed with an antibody recognizing phosphorylated GSK3 $\alpha$ (Ser 21), reprobed for GSK3 $\beta$ (Ser 9), and then reprobed again for actin as a loading control. The other blot was probed for total GSK3 (both $\alpha$ and $\beta$ isoforms) then reprobed for actin as a loading control. Results are representative of three independent experiments. (B, C) GSK3 inhibitors reduce apoptosis in response to NGF withdrawal. CB/PAx of compartmented cultures received NGF antibodies while DAx were treated with either $50 \mathrm{ng} / \mathrm{ml}$ NGF (white bars) or NGF antibodies (black bars). Lithium chloride $(15 \mathrm{mM})$ or kenpaullone $(5 \mu \mathrm{M})$ was provided to the compartments indicated during treatment. Apoptosis was assessed at $24 \mathrm{~h}$ as in Figure $1 \mathrm{C}$ and plotted ( \pm SEM, $n=9$ cultures, $* P<0.001$, one-way ANOVA post-hoc Scheffe test). Results are representative of three independent experiments. 
A

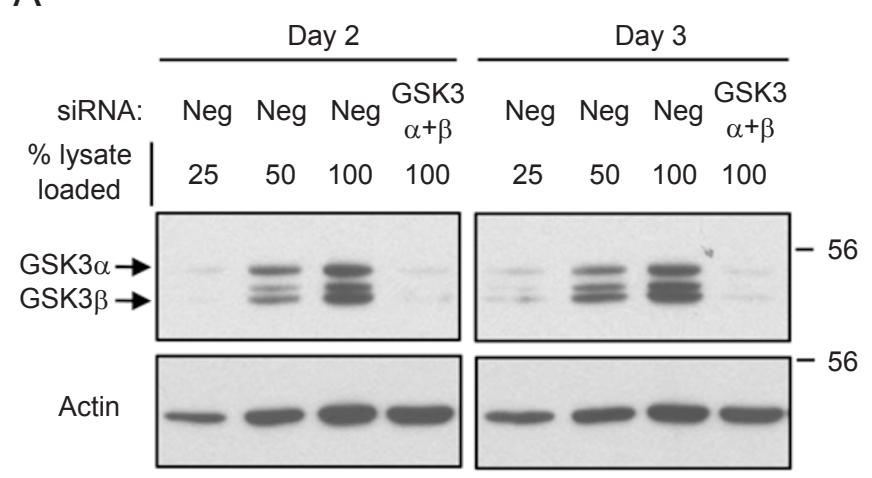

B

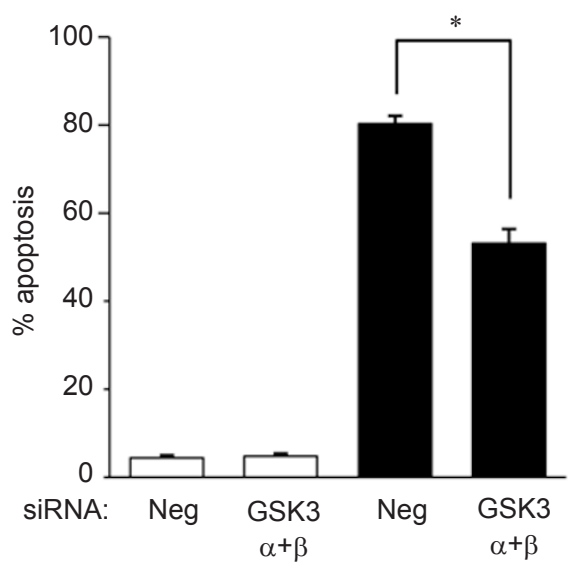

Figure 8 siRNA knockdown of GSK3 $\alpha$ and GSK3 $\beta$ decreases apoptosis in response to NGF withdrawal. Sympathetic neurons were transfected by nucleofection with siRNAs targeting rat GSK $3 \alpha$ and rat GSK3 $\beta$ mRNA $(G S K 3 \alpha+\beta)$ prior to plating in mass culture. Control transfections were also carried out with non-specific siRNA (Neg). (A) Quantitation of knockdown. Transfected neurons were harvested on day 2 or day 3 and loaded onto SDS-PAGE gels in the relative amounts indicated (10 $\mu \mathrm{g}=100 \%$ ). The immunoblot was probed with an antibody recognizing total GSK3 $\alpha$ and GSK3 $\beta$. The blot was reprobed for actin as a loading control. Results are representative of three independent experiments. (B) Apoptosis in response to NGF withdrawal. On day 2 in culture, transfected neurons were maintained in NGF (white bars) or deprived of NGF (black bars) for $24 \mathrm{~h}$. Apoptosis was quantified as above and plotted ( \pm SEM, $n=9$ cultures, compiled from three experiments, ${ }^{*} P<0.001$, oneway ANOVA post-hoc Tukey HSD).

NGF withdrawal $[17,18,38]$. Consistent with this, in our cultures, reduction of c-jun protein by siRNA decreased apoptosis of sympathetic neurons in response to NGF withdrawal (data not shown).

The initial clue that NGF withdrawal from the distal axons generates a retrograde apoptotic signal was the observation that c-jun is activated in the cell bodies by withdrawal of NGF from the distal axons, even when neuronal survival is supported by NGF supplied directly to the cell bodies and proximal axons. This was unexpected because neurons supplied with NGF at their cell bodies and proximal axons have an ample supply of NGF-induced survival signals - enough to keep them alive and produce larger NGF-induced alterations in gene expression than observed in neurons supplied with NGF only at their distal axons [39]. In the context of the strong NGF survival signaling generated at the cell bodies and proximal axons, how could the loss of NGF survival signaling from the distal axons produce nuclear accumulation of c-jun as robust and persistent as seen in neurons completely deprived of NGF? And if it did, why wasn't the nuclear accumulation of c-jun sufficient to initiate apoptosis and kill the neurons? It seems more likely that NGF deprivation at the distal axons produces an apoptotic retrograde signal that fully activates c-jun in the cell bodies which initiates the apoptotic program, but that survival signaling generated by NGF applied to cell bodies/proximal axons blocks the apoptotic program downstream of c-jun. We further tested this hypothesis by blocking axonal transport in the distal axons with colchicine which strongly inhibited the activation of cjun in the cell bodies of NGF-deprived neurons. Since the activation of c-jun was dependent upon axonal transport in NGF-deprived distal axons, it appears that a signal traveling retrogradely in the axons was responsible for activating c-jun rather than the loss of a signal that had been traveling retrogradely in the axons when they were supplied with NGF. A pro-apoptotic signal traveling retrogradely at a velocity of $10-20 \mathrm{~mm} / \mathrm{h}$ [40] could traverse the full length of the axons in our cultures in less than an hour which is well within the 3-6 h time frame of c-jun activation during NGF withdrawal [16]. Colchicine applied to the distal axons caused their disintegration after $12 \mathrm{~h}$ of treatment (data not shown). Therefore, we were unable to determine if $24 \mathrm{~h}$ treatment with colchicine could prevent apoptosis in NGF-deprived neurons since disintegration would abrogate signaling from the original distal axons, and the remaining axon stumps could possibly send injury signals that stimulate apoptosis.

It should be noted that in the novel mechanism presented, neither NGF nor TrkA need to be retrogradely transported to support neuronal survival which is consistent with the results of previous studies from our laboratory [10-12]. NGF can support neuronal survival by functioning locally in the distal axons to suppress the 
retrograde apoptotic signal that triggers c-jun activation required for apoptosis. It is also interesting that once generated in the distal axons, the retrograde apoptotic signal that activates c-jun is not turned off by passage into proximal axons and cell bodies supplied with NGF. This suggests that once it is turned on by the loss of NGF signaling, the retrograde apoptotic signal cannot be turned off by the restoration of NGF signaling.

Our hypothesis that NGF deprivation of the distal axons gives rise to a retrograde apoptotic signal is supported by our identification of pharmacological agents, rottlerin and chelerythrine, that block the retrograde induction of c-jun and apoptosis when applied to distal axons of NGF-deprived neurons, but not when applied to cell bodies and proximal axons. If these kinase inhibitors work by activating a survival signaling mechanism, they should, like NGF, also be able to support survival when applied to the cell bodies and proximal axons. The fact that they work only from the distal axons is consistent with the hypothesis that they block the generation of a retrograde apoptotic signal that is generated in NGFdeprived distal axons. Our results showing that rottlerin's protective actions are localized to the distal axon segments containing axon terminals, and not to intermediate axon segments, suggest that the retrograde apoptotic signal may be generated in or near the axon terminals.

PKC $\delta$ activity is inhibited by both rottlerin and chelerythrine; however, siRNA knockdown of PKC $\delta$ did not prevent apoptosis or prevent rottlerin from blocking apoptosis of NGF-deprived neurons. We cannot rule out a role for PKC $\delta$ since the residual PKC $\delta$ in transfected neurons could possibly have been sufficient to generate the apoptotic signal, but the lack of effect of knocking down PKC $\delta$ suggests that rottlerin and chelerythrine may produce their effects by inhibiting a different molecule.

We found that NGF deprivation of distal axons activates (dephosphorylates) GSK3 in distal axons which is blocked by rottlerin applied to distal axons but not by rottlerin applied to cell bodies and proximal axons. These results are consistent with a scenario in which NGF deprivation activates GSK3 by a rottlerin-sensitive mechanism in the distal axons. We found that lithium chloride and kenpaullone, which directly inhibit GSK3 activity, both prevented apoptosis when applied to either distal axons or cell bodies/proximal axons which is consistent with a possible role for activated GSK3 in carrying a retrograde apoptotic signal along the axon. Rottlerin applied to distal axons may have produced a slight increase in the phosphorylation of GSK3 in cell bodies/proximal axons (Figure 7A, compare lanes 2 and 3) which may be a result of constitutive retrograde transport of phosphorylated, inactivated GSK3 from the distal ax- ons supplied with NGF switching to retrograde transport of dephosphorylated, activated GSK3 when the axons were deprived of NGF. However, if GSK3 is transported as a consequence of its activation, this may produce little or no change in the level of phosphorylated GSK3 in the cell bodies/proximal axons. Therefore, the small changes in phosphorylation of GSK3 in the cell bodies/proximal axons are difficult to interpret at this time.

Unlike our results with knockdown of $\mathrm{PKC} \delta$, partial knockdown of GSK3 protein levels resulted in a decrease in NGF deprivation-induced apoptosis. It is plausible that the inhibition of apoptosis in siRNA-transfected neurons is incomplete due to insufficient knockdown of GSK3. Although immunoblot analysis suggests at least a $75 \%$ reduction in GSK3 protein levels, we do not know whether all neurons have a $75 \%$ or greater decrease in GSK3 or if there is a large variation in reduction of GSK3 from neuron to neuron. If there is a variation in GSK3 levels within the neuronal population, only those with loss of GSK3 sufficient to prevent the generation/ maintenance of the apoptotic signal would be protected. The results with GSK3 clearly represent the initial groundwork for biochemically defining the retrograde apoptotic signaling system. Other possible candidates for carrying the retrograde apoptotic signal include members of the Stress-Activated Protein Kinase (SAPK) pathway, which have been partially characterized in sympathetic neurons [41-44] and lie directly upstream of c-jun, or cjun itself.

Importantly, we do not see inhibition of the retrograde apoptotic signal generated in distal axons/terminals as the only mechanism of retrograde survival support by neurotrophins or as mutually exclusive with other possible mechanisms that may be operating within the same neuron. NGF-TrkA complexes can be retrogradely transported and activate positive survival signaling in the cell bodies, a process that is the basis of the "signaling endosome hypothesis" [8]. Our previous work has shown that NGF can activate retrograde survival signals in the distal axons that are downstream of TrkA activation, which support neuronal survival without the concurrent retrograde transport of NGF complexed to activated TrkA [10-12].

It should be noted that TrkA is not the only neurotrophin receptor present in sympathetic neurons. p75NTR is a neurotrophin receptor that has been implicated in promoting the apoptosis of sympathetic neurons in vivo [45]. It has been proposed in several studies that $\mathrm{p} 75 \mathrm{~N}-$ TR activation generates pro-apoptotic signals that are suppressed by TrkA signaling and that during development neurons receiving insufficient TrkA signaling due to limiting amounts of NGF are susceptible to p75NTR 
death signaling [46, 47]. The neurotrophins, BDNF and NT-4, two of the ligands of p75NTR, are expressed in sympathetic neurons, and it is proposed on the basis of mathematical modeling that their secretion may lead to paracrine signaling that promotes apoptosis of neurons in the context of insufficient TrkA signaling [47]. It is parsimonious to think that the axonal apoptotic signal may be a neurotrophin-activated, p75NTR-based signal, however, BDNF and NT-4 only promote apoptosis when they activate p75NTR at the cell bodies/proximal axons [47]. Moreover, in all of the published work of which we are aware, BDNF or NT-4 must be exogenously added to the medium to stimulate p75NTR-mediated apoptosis in cultured sympathetic neurons. Therefore, the axonal apoptotic signal, we describe, represents a distinct pathway.

What are the possible functions that may be served by a retrograde apoptotic signal generated in NGF-deprived axons? One possibility is that this mechanism may produce a faster apoptotic response than can be achieved, without waiting for survival signals produced by NGF to dissipate. Since the half-life of NGF in sympathetic neurons in compartmented cultures is about $6 \mathrm{~h}$ [40], and if a significant fraction of internalized NGF is bound to TrkA in signaling endosomes, which continue to signal until they reach the cell bodies, it is hard to see how the level of NGF in the neurons could be reduced enough after 6 $\mathrm{h}$ of NGF deprivation to produce the dramatic accumulation of activated c-jun in the nuclei. The time required to turn over NGF would be even greater in neurons with longer axons. Also, NGF retrograde survival signaling has numerous downstream effects, including activation of pro-survival signaling molecules and changes in gene expression, that result in production of proteins with anti-apoptotic effects $[48,49]$. Considerable time may be required to inhibit or degrade these proteins after termination of TrkA signaling. Perhaps the axonal apoptotic signal serves to hasten apoptosis by activating an apoptotic pathway that quickly bypasses residual survival signals to kill the neuron quickly.

Another possible function is that a retrograde apoptotic signal could allow neurons to compare death and survival signals coming from different axon branches. If a neuron had part of its axon terminal branches located in an appropriate target that supplies enough NGF-induced retrograde survival signals to keep the neuron alive, but had other axon terminal branches localized in inappropriate targets that supply little or no NGF, it might be beneficial to the organism to override the survival signals and eliminate the neuron entirely. Thus, a retrograde proapoptotic signal generated in NGF-deprived axons could be part of a mechanism whereby the relative magnitude of death and survival signals that are being received from the axons determine the survival of the neuron.

There is no reason to assume that retrograde apoptotic signaling systems operate exclusively in sympathetic neurons during NGF-dependent development. The axonal apoptotic signal may continue to be employed in adult neurons to alert the cell body about axon injury, toxic conditions, and so on. Other examples of axonal apoptotic signals have been identified that contribute to HIV neuropathy and axon injury $[50,51]$. The existence of retrograde death signals in axons initiated by neurotrophin deprivation has broad implications for discovering treatments for neurodegenerative diseases and neurotrauma. Activation of the c-jun pathway has been linked to the pathology of both Alzheimer's disease and Parkinson's disease $[52,53]$. It will be important to determine how widespread retrograde axonal death signaling systems are and what other stimuli can activate them. The existence of retrograde death signaling systems in axons will certainly change the way in which we examine the life and death of neurons in future research.

\section{Materials and Methods}

\section{Sympathetic neuron cultures and treatments}

Superior cervical ganglia were isolated from 0-2-day-old Sprague-Dawley rats supplied by the University of Alberta Health Sciences Laboratory Animal Services. Compartmented cultures were constructed with a Teflon divider (Tyler Research Corporation, Edmonton, AB) on a 35-mm dish as described in Figure 1A. Three-compartment dividers have 1-mm barriers separating a 1-mm-wide center compartment from 7-mm-wide distal compartments. Five-compartment dividers have an additional 1-mm-wide intermediate compartment between the center and distal compartments. Neurons were dissociated and plated in center compartments of three- or five-compartmented cultures at a density of 0.4 ganglia per culture as previously described [54]. For mass cultures, neurons were plated in 96-well culture plates (Corning Inc., Corning, NY) at a density of 0.12 ganglia per well. Cultures were maintained in L-15 medium (Invitrogen, Carlsbad, CA) modified as described previously [54]. At plating, NGF (2.5S NGF from Alamone Laboratories, Jerusalem, Israel) was provided in distal compartments and in mass cultures at $50 \mathrm{ng} / \mathrm{ml}$ and at $10 \mathrm{ng} /$ $\mathrm{ml}$ in cell body compartments. In five-compartmented cultures, the intermediate compartments were given $15 \mathrm{ng} / \mathrm{ml} \mathrm{NGF}$. The medium provided to center compartments containing the neuronal cell bodies, and to mass cultures, contained $2.5 \%$ rat serum and $280 \mu \mathrm{M}$ vitamin C (Sigma-Aldrich, St Louis, MO). Distal- and intermediate-compartment media lacked rat serum or vitamin $\mathrm{C}$ [55]. During the first week in culture, $10 \mu \mathrm{M}$ cytosine arabinoside (Sigma-Aldrich) was supplied to center compartments and mass cultures to kill non-neuronal cells. Cultures were used in experiments between 6 and 8 days in vitro unless otherwise indicated.

During experimental treatments, mass cultures and center compartments containing cell bodies were always provided medium containing rat serum and vitamin C. Compartments were 
supplied with medium containing either $50 \mathrm{ng} / \mathrm{ml} \mathrm{NGF}$ or $24 \mathrm{nM}$ of antibodies to NGF (Cedar Lane, Hornby, ON). When center compartments were treated with NGF, the slot containing the cell bodies, as well as the dish perimeter, were filled with medium to prevent depletion of NGF during incubation. All inhibitors (Calbiochem, San Diego, CA) except $\mathrm{LiCl}$ were prepared as working stocks from powder reconstituted in DMSO: Rottlerin at $1.25 \mathrm{mM}$, Gö6976 at $12.5 \mu \mathrm{M}$, chelerythrine at $5 \mathrm{mM}$, and kenpaullone at $2.5 \mathrm{mM}$. Inhibitor stocks were added directly to medium at a dilution of 1:500 to achieve final concentrations. All media used in these experiments contained $0.2 \%$ DMSO. For $\mathrm{LiCl}$ experiments, $\mathrm{LiCl}$ (Sigma-Aldrich, St Louis, MO) was added to the media, while maintaining the original osmolarity. $\mathrm{NaCl}$ was added to all other media as a control. For experiments longer than $24 \mathrm{~h}$, the medium in all compartments was replaced every $24 \mathrm{~h}$.

\section{Neuronal survival assays}

Following experimental treatments, cultures were rinsed with phosphate-buffered saline (PBS) and then fixed with 4\% paraformaldehyde (Fisher Scientific, Ottawa, ON) in PBS for $15 \mathrm{~min}$ at room temperature. Mass cultures and the center compartments of cultures were incubated with $10 \mu \mathrm{M}$ Hoechst 33258 (Molecular Probes, Eugene, OR) in PBS for $10 \mathrm{~min}$. Stained nuclei were examined using a Leica DMIRE2 microscope (Leica, Richmond Wetzlar, Germany) with a UV filter. In cultures deprived of NGF for $24 \mathrm{~h}$, a neuron was scored apoptotic if it had a condensed nucleus. After $24 \mathrm{~h}$, nuclear condensation was followed by loss of nuclear staining in the majority of apoptotic neurons. Therefore, in cultures deprived of NGF for 48 and $72 \mathrm{~h}$, a neuron was assessed as apoptotic if it lacked a nucleus by UV fluorescence. Individual apoptotic neurons in light images were not always clearly visualized, and we estimated a $5 \%$ margin of error in quantitation at 48 and 72-h time points.

\section{Immunocytochemistry}

Compartmented cultures were fixed with $4 \%$ paraformaldehyde at room temperature for $15 \mathrm{~min}$ without removing the Teflon dividers. Cells were permeabilized with $0.2 \%$ Triton X-100 (Sigma-Aldrich) for $10 \mathrm{~min}$, then incubated in a blocking buffer of 5.5\% normal goat serum (Invitrogen) in TBS (tris-buffered saline) with $0.1 \%$ Tween-20 (TBS-T) (Fisher Scientific) for $1 \mathrm{~h}$. Phospho c-jun (Ser 63) antibody (Cell Signaling Technology, Danvers, MA), at a dilution of 1:1 500, or tubulin antibody (Sigma-Aldrich) at a dilution of 1:500, was applied to cultures in a buffer of 3\% BSA in TBS overnight at $4{ }^{\circ} \mathrm{C}$. Cultures were further processed using a rabbit IgG Vectastain Elite ABC Kit (Vector Labs, Burlingame, CA), and immunoreactivity was visualized by the peroxidase reaction of a DAB peroxidase substrate (Sigma Fast 3,3-diaminobenzidine tablet sets, Sigma-Aldrich). Images were taken with a Nikon Diaphot inverted light microscope equipped with a Nikon D70 digital camera (Nikon Canada, Mississauga, ON). The photographic montage of tubulin-stained neurons was assembled using Canon Photostitch software (Canon Canada, Mississauga, ON).

\section{Immunoblotting of compartmented cultures}

Following treatment, cultures were rinsed with ice-cold TBS containing sodium fluoride (Sigma-Aldrich, St Louis, MO) and sodium orthovanadate. For CB/PAx compartments, $10 \mu \mathrm{l}$ of $3 \times$ sample buffer was applied to each culture, and cellular material was dislodged from the substratum by scraping with a hand-held, gelloading style micropipette tip, then harvested with a micropipette and pooled in a single sample per group. For distal compartments, each culture was harvested sequentially in $30 \mu \mathrm{l}$ of $3 \times$ sample buffer by scraping using a $200 \mu$ pipette tip, to keep extract volumes low. Material from five cultures was pooled for each group. Extracts were boiled for $5 \mathrm{~min}$, resolved on SDS-PAGE gels, and transferred to Immobilon-P PVDF membranes (Millipore, Billerica, MA). Antibodies recognizing caspase 3 (p35 and p17, 1:1 000), phosphorylated c-jun (Ser 63, 1:1 000), phospho-GSK3 $\alpha$ (Ser 21, 1:1 000), and phospho-GSK3 $\beta$ (Ser 9, 1:3 000) were purchased from Cell Signaling Technology. Antibodies recognizing actin (1:2 $000)$ and $\beta$-tubulin (1:2 000) were purchased from Sigma-Aldrich. Immunoblots were probed overnight with antibodies at the concentration indicated, and immunoreactivity was detected using enhanced chemiluminescence (SuperSignal West Femto Maximum Sensitivity Substrate, Pierce, Rockford, IL).

\section{siRNA transfection and treatments}

Pools of siRNAs or individual siRNAs purchased from Dharmacon (Lafayette, CO) were used to target the rat mRNA sequences of PKC $\delta$ (SMARTpool siRNA, cat\# M-080142-00), GSK3 $\alpha$ (ON-TARGETplus siRNA duplex, cat\# J-080107-09 and J-080107-11), and GSK3 $\beta$ (ON-TARGETplus SMARTpool siRNA, cat\# L-080108-00). Non-targeting siRNA (siCONTROL \#1, Dharmacon) was used as a negative control. $200 \mu \mathrm{M}$ stock solutions of pools/individual siRNAs were made in suspension buffer provided by the manufacturer and used at a final concentration of $18 \mu \mathrm{M}$. Superior cervical ganglia were isolated and dissociated normally. Dissociated neurons were transfected using a Nucleofector electroporation apparatus (Amaxa, Cologne, Germany) as described by He et al. [27] modified from Hasaka et al. [56]. Briefly, dissociated cells (about 1 million) were pelleted at $800 \times g$ for $5 \mathrm{~min}$ and resuspended in $100 \mu \mathrm{l}$ of Rat Neuron Nucleofection solution (Amaxa). The cells were then added to a transfection cuvette (Amaxa) containing $10 \mu$ of RNA suspension buffer or siRNA. Cells were transfected on setting G-13, transferred immediately to a warm plating medium (L-15 with $50 \mathrm{ng} / \mathrm{ml} \mathrm{NGF}$ and rat serum), and plated at a density of 2 ganglia per well (24-well plate) and 0.4 ganglia per well (96-well plate). The plating medium was replaced with a normal culture medium at $16 \mathrm{~h}$, and neurons were grown as described above for mass cultures.

For immunoblotting, 1 well was harvested in a lysis buffer containing protease inhibitors (Complete Mini, Roche, Mannheim, Germany), sodium fluoride (Sigma-Aldrich), and sodium orthovanadate (Sigma-Aldrich) on ice for $30 \mathrm{~min}$. Equivalent amounts of protein for each sample were resolved on 10\% SDS-PAGE gels. Immunoblots were probed for PKC $\delta$ (1:2 000 Santa-Cruz), phosphorylated c-jun (1:1 000, Cell Signaling Technology), total GSK3 ( $\alpha$ and $\beta$ isoform, 1:1 000, Calbiochem), $\beta$-tubulin (1:5 000, Sigma-Aldrich), or actin (1:2 000, Sigma-Aldrich) as indicated. Immunoreactivity was detected using enhanced chemiluminescence (Pierce).

\section{Conjugation of Cy3 to NGF and immunofluorescence imag- ing}

Lyophilized NGF $(250 \mu \mathrm{g})$ was dissolved in $250 \mu \mathrm{l}$ of sodium carbonate buffer, $\mathrm{pH} 9.3$, and then mixed with half a pack of $\mathrm{Cy} 3$ mono-reactive NHS Esters dye (Amersham) pre-dissolved in 500 
$\mu l$ of the same buffer. The reaction was incubated at room temperature for $60 \mathrm{~min}$. The solution was purified with a NAP-5 gel filtration column (Amersham) equilibrated with a sodium phosphate buffer, $\mathrm{pH} 7.0$, to remove the free $\mathrm{Cy} 3$. The concentration of the purified Cy3-NGF was determined according to the manufacturer's directions (Amersham). The Cy3-NGF was applied to compartmented cultures as described for non-labeled NGF. A Leica DMIRE2 microscope was used to visualize Cy3 fluorescence.

\section{Acknowledgments}

We thank Norma Jean Valli, Department of Cell Biology, University of Alberta, for her excellent technical assistance. We thank Dr Thomas Simmen, Department of Cell Biology, University of Alberta, for use of his Amaxa Nucleofector. This research was supported by the Canadian Institutes of Health Research.

\section{References}

1 Levi-Montalcini R, Angeletti PU. Nerve growth factor. Physiol Rev 1968; 48:534-569.

2 Oppenheim RW. Cell death during development of the nervous system. Annu Rev Neurosci 1991; 14:453-501.

3 Huang EJ, Reichardt LF. Neurotrophins: roles in neuronal development and function. Annu Rev Neurosci 2001; 24:677736.

4 Hendry IA, Stockel K, Thoenen H, Iversen LL. The retrograde axonal transport of nerve growth factor. Brain Res 1974; 68:103-121.

5 Hendry IA. The effect of the retrograde axonal transport of nerve growth factor on the morphology of adrenergic neurones. Brain Res 1977; 134:213-223.

6 Ehlers MD, Kaplan DR, Price DL, Koliatsos VE. NGF-stimulated retrograde transport of trkA in the mammalian nervous system. J Cell Biol 1995; 130:149-156.

7 Delcroix JD, Valletta JS, Wu C, et al. NGF signaling in sensory neurons: evidence that early endosomes carry NGF retrograde signals. Neuron 2003; 39:69-84.

8 Howe CL, Mobley WC. Long-distance retrograde neurotrophic signaling. Curr Opin Neurobiol 2005; 15:40-48.

9 Zweifel LS, Kuruvilla R, Ginty DD. Functions and mechanisms of retrograde neurotrophin signalling. Nat Rev Neurosci 2005; 6:615-625.

10 MacInnis BL, Campenot RB. Retrograde support of neuronal survival without retrograde transport of nerve growth factor. Science 2002; 295:1536-1539.

11 Mok SA, Campenot RB. A nerve growth factor-induced retrograde survival signal mediated by mechanisms downstream of TrkA. Neuropharmacology 2007; 52:270-278.

12 MacInnis BL, Senger DL, Campenot RB. Spatial requirements for TrkA kinase activity in the support of neuronal survival and axon growth in rat sympathetic neurons. Neuropharmacology 2003; 45:995-1010.

13 Ham J, Eilers A, Whitfield J, Neame SJ, Shah B. c-Jun and the transcriptional control of neuronal apoptosis. Biochem Pharmacol 2000; 60:1015-1021.

14 Hibi M, Lin A, Smeal T, Minden A, Karin M. Identification of an oncoprotein- and UV-responsive protein kinase that binds and potentiates the c-Jun activation domain. Genes Dev 1993; 7:2135-2148.

15 Derijard B, Hibi M, Wu IH, et al. JNK1: a protein kinase stimulated by UV light and Ha-Ras that binds and phosphorylates the c-Jun activation domain. Cell 1994; 76:1025-1037.

16 Virdee K, Bannister AJ, Hunt SP, Tolkovsky AM. Comparison between the timing of JNK activation, c-Jun phosphorylation, and onset of death commitment in sympathetic neurones. $J$ Neurochem 1997; 69:550-561.

17 Whitfield J, Neame SJ, Paquet L, Bernard O, Ham J. Dominant-negative c-Jun promotes neuronal survival by reducing BIM expression and inhibiting mitochondrial cytochrome c release. Neuron 2001; 29:629-643.

18 Palmada M, Kanwal S, Rutkoski NJ, et al. c-jun is essential for sympathetic neuronal death induced by NGF withdrawal but not by p75 activation. J Cell Biol 2002; 158:453-461.

19 Tsui-Pierchala BA, Ginty DD. Characterization of an NGFP-TrkA retrograde-signaling complex and age-dependent regulation of TrkA phosphorylation in sympathetic neurons. $J$ Neurosci 1999; 19:8207-8218.

20 Heerssen HM, Pazyra MF, Segal RA. Dynein motors transport activated Trks to promote survival of target-dependent neurons. Nat Neurosci 2004; 7:596-604.

21 Gschwendt M, Muller HJ, Kielbassa K, et al. Rottlerin, a novel protein kinase inhibitor. Biochem Biophys Res Commun 1994; 199:93-98.

22 Herbert JM, Augereau JM, Gleye J, Maffrand JP. Chelerythrine is a potent and specific inhibitor of protein kinase C. Biochem Biophys Res Commun 1990; 172:993-999.

23 Martiny-Baron G, Kazanietz MG, Mischak H, et al. Selective inhibition of protein kinase $\mathrm{C}$ isozymes by the indolocarbazole Go 6976. J Biol Chem 1993; 268:9194-9197.

24 Wright KM, Vaughn AE, Deshmukh M. Apoptosome dependent caspase-3 activation pathway is non-redundant and necessary for apoptosis in sympathetic neurons. Cell Death Differ 2007; 14:625-633

25 Choi BH, Hur EM, Lee JH, Jun DJ, Kim KT. Protein kinase Cdelta-mediated proteasomal degradation of MAP kinase phosphatase-1 contributes to glutamate-induced neuronal cell death. J Cell Sci 2006; 119:1329-1340.

26 Kaul S, Kanthasamy A, Kitazawa M, Anantharam V, Kanthasamy AG. Caspase-3 dependent proteolytic activation of protein kinase $\mathrm{C}$ delta mediates and regulates 1-methyl-4phenylpyridinium (MPP+)-induced apoptotic cell death in dopaminergic cells: relevance to oxidative stress in dopaminergic degeneration. Eur J Neurosci 2003; 18:1387-1401.

27 He Y, Francis F, Myers KA, et al. Role of cytoplasmic dynein in the axonal transport of microtubules and neurofilaments. $J$ Cell Biol 2005; 168:697-703.

28 Ahmed S, Shibazaki M, Takeuchi T, Kikuchi H. Protein kinase Ctheta activity is involved in the 2,3,7,8-tetrachlorodibenzo-pdioxin-induced signal transduction pathway leading to apoptosis in L-MAT, a human lymphoblastic T-cell line. FEBS J 2005; 272:903-915.

29 Beurel E, Jope RS. The paradoxical pro- and anti-apoptotic actions of GSK3 in the intrinsic and extrinsic apoptosis signaling pathways. Prog Neurobiol 2006; 79:173-189.

30 Garrido JJ, Simon D, Varea O, Wandosell F. GSK3 alpha and GSK3 beta are necessary for axon formation. FEBS Lett 2007; 
581:1579-1586.

31 Kim WY, Zhou FQ, Zhou J, et al. Essential roles for GSK$3 \mathrm{~s}$ and GSK-3-primed substrates in neurotrophin-induced and hippocampal axon growth. Neuron 2006; 52:981-996.

32 Hooper C, Killick R, Lovestone S. The GSK3 hypothesis of Alzheimer's disease. J Neurochem 2008; 104:1433-1439.

33 Woodgett JR. Molecular cloning and expression of glycogen synthase kinase-3/factor A. Embo J 1990; 9:2431-2438.

34 Sutherland C, Cohen P. The alpha-isoform of glycogen synthase kinase- 3 from rabbit skeletal muscle is inactivated by p70 S6 kinase or MAP kinase-activated protein kinase-1 in vitro. FEBS Lett 1994; 338:37-42.

35 Sutherland C, Leighton IA, Cohen P. Inactivation of glycogen synthase kinase-3 beta by phosphorylation: new kinase connections in insulin and growth-factor signalling. Biochem $J$ 1993; 296:15-19.

36 Bain J, Plater L, Elliott M, et al. The selectivity of protein kinase inhibitors: a further update. Biochem J 2007; 408:297315.

37 Campenot RB, MacInnis BL. Retrograde transport of neurotrophins: fact and function. J Neurobiol 2004; 58:217-229.

38 Ham J, Babij C, Whitfield J, et al. A c-Jun dominant negative mutant protects sympathetic neurons against programmed cell death. Neuron 1995; 14:927-939.

39 Toma JG, Rogers D, Senger DL, Campenot RB, Miller FD. Spatial regulation of neuronal gene expression in response to nerve growth factor. Dev Biol 1997; 184:1-9.

40 Ure DR, Campenot RB. Retrograde transport and steady-state distribution of 125I-nerve growth factor in rat sympathetic neurons in compartmented cultures. J Neurosci 1997; 17:12821290.

41 Eilers A, Whitfield J, Shah B, et al. Direct inhibition of c-Jun $\mathrm{N}$-terminal kinase in sympathetic neurones prevents c-jun promoter activation and NGF withdrawal-induced death. J Neurochem 2001; 76:1439-1454.

42 Bazenet CE, Mota MA, Rubin LL. The small GTP-binding protein $\mathrm{Cdc} 42$ is required for nerve growth factor withdrawalinduced neuronal death. Proc Natl Acad Sci USA 1998; 95:3984-3989.

43 Harding TC, Xue L, Bienemann A, et al. Inhibition of JNK by overexpression of the JNL binding domain of JIP-1 prevents apoptosis in sympathetic neurons. J Biol Chem 2001; 276:4531-4534.

$44 \mathrm{Xu}$ Z, Maroney AC, Dobrzanski P, Kukekov NV, Greene LA. The MLK family mediates c-Jun N-terminal kinase activation in neuronal apoptosis. Mol Cell Biol 2001; 21:4713-4724.

45 Bamji SX, Majdan M, Pozniak CD, et al. The p75 neurotrophin receptor mediates neuronal apoptosis and is essential for naturally occurring sympathetic neuron death. $J$ Cell Biol 1998; 140:911-923.

46 Majdan M, Walsh GS, Aloyz R, Miller FD. TrkA mediates developmental sympathetic neuron survival in vivo by silencing an ongoing p75NTR-mediated death signal. J Cell Biol 2001; 155:1275-1285.

47 Deppmann CD, Mihalas S, Sharma N, et al. A model for neuronal competition during development. Science 2008; 320:369373.

48 Crowder RJ, Freeman RS. Phosphatidylinositol 3-kinase and Akt protein kinase are necessary and sufficient for the survival of nerve growth factor-dependent sympathetic neurons. $J \mathrm{Neu}$ rosci 1998; 18:2933-2943.

49 Riccio A, Ahn S, Davenport CM, Blendy JA, Ginty DD. Mediation by a CREB family transcription factor of NGF-dependent survival of sympathetic neurons. Science 1999; 286:23582361.

50 Melli G, Keswani SC, Fischer A, Chen W, Hoke A. Spatially distinct and functionally independent mechanisms of axonal degeneration in a model of HIV-associated sensory neuropathy. Brain 2006; 129:1330-1338.

51 Carson C, Saleh M, Fung FW, Nicholson DW, Roskams AJ. Axonal dynactin p150Glued transports caspase- 8 to drive retrograde olfactory receptor neuron apoptosis. J Neurosci 2005; 25:6092-6104.

52 Okazawa H, Estus S. The JNK/c-Jun cascade and Alzheimer's disease. Am J Alzheimers Dis Other Demen 2002; 17:79-88.

53 Peng J, Andersen JK. The role of c-Jun N-terminal kinase (JNK) in Parkinson's disease. IUBMB Life 2003; 55:267-271.

54 Campenot RB, Martin G. Construction and use of compartmented cultures for studies of cell biology of neurons. In: Federoff S, Richardson A, eds. Protocols for Neural Cell Culture. Totowa, NJ: Humana Press, 2001:49-57.

55 Campenot RB. Development of sympathetic neurons in compartmentalized cultures. II. Local control of neurite survival by nerve growth factor. Dev Biol 1982; 93:13-21.

56 Hasaka TP, Myers KA, Baas PW. Role of actin filaments in the axonal transport of microtubules. J Neurosci 2004; 24:1129111301.

(Supplementary information is linked to the online version of the paper on the Cell Research website.) 